MS16-P09

\section{Titanyl sulfate dihydrate - template for preparation of 1D titania structures}

Mariana Klementova $^{1}$, Monika Motlochova ${ }^{2}$, Jaroslav Kupcik ${ }^{2}$, Lukas Palatinus ${ }^{1}$, Eva Plizingrova ${ }^{2}$, Lorant Szatmary ${ }^{3}$, Jan Subrt ${ }^{2}$

1. Institute of Physics of the Czech Academy of Sciences, Prague, Czech Republic

2. Institute of Inorganic Chemistry of the CAS, v.v.i., CZ-250 68 Husinec-Rez, Husinec-Rez, Czech Republic

3. Nuclear Research Institute, a.s., Hlavní 130, CZ-250 68 HusinecRez, Husinec-Rez, Czech Republic

email: klemari@fzu.cz

Titanyl sulfate dihydrate (TSD, $\left.\mathrm{TiOSO}_{4} \cdot 2 \mathrm{H}_{2} \mathrm{O}\right)$ can serve as a template for preparation of 1D titania structures. When immersed in aqueous ammonia at a temperature of about 0 ${ }^{\circ} \mathrm{C}$ the crystals of TSD provide solid residue composed of particles, whose shape and dimensions correspond perfectly to the rod-like particle morphology of the initial titanyl sulfate with composition matching that of metatitanic acid. When annealed up to $1200{ }^{\circ} \mathrm{C}$, the rod-like morphology of particles is retained, while the phase composition changes to anatase/rutile. To understand the mechanism of the phase transformation that is not accompanied with a change of particle shape, the structure of TSD had to be determined.

The TSD crystals form very thin platelets unsuitable for investigation by single-crystal $\mathrm{x}$-ray diffraction. The structure was therefore solved using dynamical refinement of precession electron diffraction tomography data using PETS [1] and Jana2006 [2].

The structure is monoclinic $P n$ with lattice parameters $a=$ 5.54(2) $\AA, b=16.77(9) \AA, c=18.74(10) \AA, b=90.5(3)^{\circ}$. 54 independent non-hydrogen atoms were easily identified in the electrostatic potential distributionobtained ab initio by charge flipping. To keep charge balance such model requires 24 hydrogen atoms, whose positions were estimated based on the bond-valence calculations [3]. 17 hydrogens out of 24 could be matched to the maxima in the difference map calculated from combined refinement of 6 datasets. The remaining hydrogen positions were estimated based on the knowledge of water molecule geometry. The agreement factor $R_{o b s}$ decreased from $10.36 \%$ to $9.89 \%$ after including the hydrogens.

The TSD structure is composed of two symmetry independent helices of corner sharing $\mathrm{TiO}_{6}$ octahedra extending along a (Figure 1). Each pseudotrigonal helix is held together by $\mathrm{SO}_{4}$ tetrahedra that share corners with odd/even $\mathrm{TiO}_{6}$ octahedra. Both hydrogens in each water molecule form hydrogen bonds toward oxygens of two different $\mathrm{SO}_{4}$ tetrahedra. One $\mathrm{H}$-bond points to the oxygen of $\mathrm{SO}_{4}$ tetrahedra from the same helix, while the other $\mathrm{H}$-bond points to the oxygen of $\mathrm{SO}_{4}$ tetrahedra from the neighboring helix. Based on the structure a tranformation mechanism could be proposed. During the reaction the sulfate anion are replaced with $\mathrm{OH}-$. The replacement of sulfate by $\mathrm{OH}-$ anions in the helix does not cause a collapse of the helices as the newly bonded $\mathrm{OH}$-groups repel one another. However, the interaction between the helices is weakened, resulting in polymer-like chains of $\mathrm{TiO}_{6}$ octahedra characteristic for the amorphous metatitanic acid.
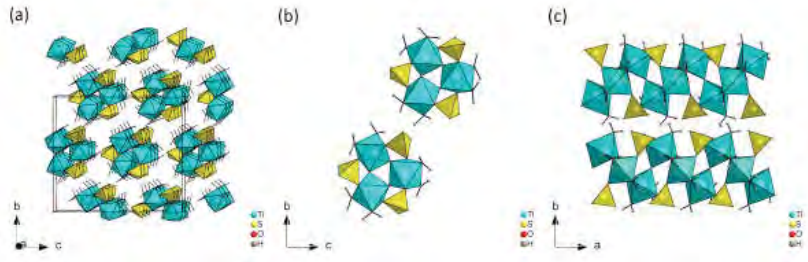

References:

[1] L. Palatinus (2011). PETS - program for analysis of electron diffraction data. Prague: Institute of Physics of the AS CR. http:// pets.fzu.cz/.

[2] V. Petř́íček, M. Dušek, L. Palatinus, Crystallographic Computing System JANA2006: General features, Z. Krist. 229-5 (2014) 345-352.

[3] I. D. Brown, The Chemical Bond in Inorganic Chemistry: The Bond Valence Model (Oxford Univ. Press, 2002).

Keywords: electron diffraction, titanyl sulfate dihydrate, pseudomorphs 